\title{
Frontières
}

\section{Les préalables à un débat sensé sur l'euthanasie, et la pertinence du document de la Commission de Réforme du droit sur l'euthanasie, considérant le projet de loi C-384}

\section{Michel T. Giroux}

Volume 24, numéro 1-2, automne 2011, printemps 2012

L’aide médicale à mourir

URI : https://id.erudit.org/iderudit/1013081ar

DOI : https://doi.org/10.7202/1013081ar

Aller au sommaire du numéro

Éditeur(s)

Université du Québec à Montréal

ISSN

1916-0976 (numérique)

Découvrir la revue

Citer cet article

Giroux, M. T. (2011). Les préalables à un débat sensé sur l'euthanasie, et la pertinence du document de la Commission de Réforme du droit sur l'euthanasie, considérant le projet de loi C-384. Frontières, 24(1-2), 18-30. https://doi.org/10.7202/1013081ar
Résumé de l'article

La Commission de réforme du droit du Canada (CRD) a publié, en 1982, un document de travail intitulé Euthanasie, aide au suicide et interruption de traitement. En 2009, la députée Francine Lalonde a déposé à la Chambre des communes le projet de loi C-384 intitulé Loi modifiant le Code criminel (droit de mourir dignement). Ce projet de loi a été défait. Une période de près de trente ans sépare ces deux documents. Quelles sont les préoccupations que partagent ces documents ? Le projet de loi envisage-t-il l'aide au suicide d'une manière qui diffère de celle du document de la CRD ? L'examen du document de la CRD, du projet de loi C-384 et des travaux de la Chambre des communes fait ressortir des difficultés persistantes dans nos discussions publiques. Certaines suggestions s'imposent à propos du débat souhaitable dans une matière aussi contentieuse que l'euthanasie et l'aide au suicide. 


\section{Résumé}

La Commission de réforme du droit du Canada (CRD) a publié, en 1982, un document de travail intitulé Euthanasie, aide au suicide et interruption de traitement. En 2009, la députée Francine Lalonde a déposé à la Chambre des communes le projet de loi C-384 intitulé Loi modifiant le Code criminel (droit de mourir dignement). Ce projet de loi a été défait. Une période de près de trente ans sépare ces deux documents. Quelles sont les préoccupations que partagent ces documents? Le projet de loi envisage-t-il l'aide au suicide d'une manière qui diffère de celle $d u$ document de la CRD ? L'examen du document de la CRD, du projet de loi C-384 et des travaux de la Chambre des communes fait ressortir des difficultés persistantes dans nos discussions publiques. Certaines suggestions s'imposent à propos du débat souhaitable dans une matière aussi contentieuse que l'euthanasie et I'aide au suicide.

Mots clés: discussion publique euthanasie - aide au suicide interruption de traitement-droit de mourir dans la dignité - Commission de réforme du droit du Canada projet de loi C-384.

\section{Abstract}

The Law Reform Commission of Canada (LRC) published, in 1982, a working paper, Euthanasia, Aiding Suicide and Cessation of Treatment. In 2009, MP Francine Lalonde proposed to the House of Commons bill C-384, An Act to amend the Criminal Code (right to die with dignity). This bill was undone. A period of about thirty years separates these two documents. What are the concerns which share these documents? Does the bill understand assisted suicide in a way which differs from that of the LRC document? The examination of the LRC document, bill C-384 and the works of the House of Commons highlights persistent difficulties in our public discussions. Some suggestions need to be done about the desirable debate on a subject as contentious as euthanasia and aiding suicide.

Keywords: public discussion euthanasia - aiding suicide - cessation of treatment - right to die with dignity - Law Reform Commission of Canada - bill C-384.

\section{LES PRÉALABLES À UN DÉBAT SENSÉ SUR L'EUTHANASIE, ET LA PERTINENCE DU DOCUMENT DE LA COMMISSION DE RÉFORME DU DROIT
SUR L'EUTHANASIE, CONSIDÉRANT
LE PROJET DE LOI C-384}

Michel T. Giroux, LL.B., M. A., Ph. D., directeur, Institut de consultation et de recherche en éthique et en droit (ICRED).

La Commission de réforme du droit du Canada (CRD) a publié, en 1982, un document de travail intitulé Euthanasie, aide au suicide et interruption de traitement. Le 13 mai 2009, madame Francine Lalonde, députée de La Pointe-de-l'Île, a déposé à la Chambre des communes le projet de loi C-384 intitulé Loi modifiant le Code criminel (droit de mourir dignement). Ce projet de loi a été défait en seconde lecture le 21 avril 2010. Il n'a donc pas fait l'objet des travaux d'un comité parlementaire. Une période de près de trente ans sépare ces deux documents, dont chacun a été rédigé dans un contexte social et juridique distinct. L'objet de ce texte est de présenter le document de la CRD et le projet de loi C-384 pour identifier les préoccupations qu'ils partagent, et pour évaluer si le projet de loi envisage l'aide au suicide d'une manière qui diffère de celle du document de la CRD. Nous compléterons ce propos par quelques remarques sur le forum de discussion approprié - judiciaire ou parlementaire - pour les questions dont nous discutons ici.

L'examen rapproché du document de la CRD, du projet de loi C-384 et des travaux de la Chambre des communes fait ressortir des difficultés persistantes dans nos discussions publiques. C'est pourquoi, avant de présenter le document de la CRD et le projet de loi C-384, certaines suggestions s'imposent à propos du débat souhaitable dans une matière aussi contentieuse que l'euthanasie et l'aide au suicide.

\section{LES PRÉALABLES \\ À UN DÉBAT SENSÉ}

Dans ce texte, les préalables représentent les conditions de nature conceptuelle sans lesquelles nous ne pouvons pas soutenir un débat judicieux, marqué par la raison. Les discussions portant sur l'euthanasie et l'aide au suicide achoppent sur trois obstacles récurrents: l'imprécision du vocabulaire employé, l'évitement de la circonstance limite et l'absence de distinction entre la règle de droit et les situations 
LES DISCUSSIONS PORTANT SUR L'EUTHANASIE ET L'AIDE AU SUICIDE ACHOPPENT SUR TROIS OBSTACLES RÉCURRENTS: L'IMPRÉCISION DU VOCABULAIRE EMPLOYÉ, L'ÉVITEMENT DE LA CIRCONSTANCE LIMITE ET L'ABSENCE DE DISTINCTION ENTRE LA RÈGLE DE DROIT ET LES SITUATIONS PARTICULIÈRES.

particulières. L'imprécision du vocabulaire employé vise ce que les interlocuteurs ont en tête lorsqu'ils utilisent le mot « euthanasie». L'évitement de la circonstance limite se produit lorsque la dispensation de soins palliatifs constitue la réponse automatique et universelle à toutes les formes de souffrance en fin de vie. Finalement, l'absence de distinction entre la règle de droit et les situations particulières dissimule la possibilité bien réelle d'un conflit irréductible entre la règle de droit et le choix qu'exprime une conscience individuelle.

\section{DES DÉFINITIONS DE L'EUTHANASIE}

Au Canada, le mot «euthanasie» ne représente aucun concept juridique. Le Code criminel ne contient aucune infraction s'appelant euthanasie; les catégories juridiques pertinentes sont l'homicide, le meurtre et l'aide au suicide. Le droit ne nous offre donc aucune définition notoire de l'euthanasie à laquelle nous pourrions nous reporter dans nos débats. L'absence d'une définition notoire contribue sans doute à la confusion observée à propos de concepts comme la cessation de traitement, l'abandon thérapeutique, la sédation terminale et l'euthanasie ${ }^{1}$.

La consultation de divers ouvrages révèle à quel point le mot «euthanasie» est susceptible de revêtir des significations divergentes. Débutons par l'étymologie du mot. Un dictionnaire historique nous la rappelle ainsi. Euthanasie « est un emprunt (1771) au grec tardif euthanasia "mort douce et facile", de $e u$ - "bien", et d'un dérivé de thanatos "mort" »(dictionnaire Le Robert, 1998: 1343). Pris dans un sens proche de son étymologie, «euthanasie» signifie l'art de rendre la mort douce.

Lorsqu'il aborde le sujet de l'euthanasie, le philosophe Martin Blais rappelle la nécessité de la définir et il propose une définition proche de l'étymologie du mot:

Pour voir un peu plus clair dans le problème de l'euthanasie, il importe d'abord de définir l'euthanasie comme telle avant de lui accoler les qualificatifs d'usage: directe, indirecte, active, passive. Comme telle, l'euthanasie devrait évoquer des moyens de soulager les souffrances d'une personne atteinte d'une maladie dont l'issue fatale est imminente.

Ainsi entendue, l'euthanasie est conforme à la morale, même si la vie est abrégée: c'est l'acte à double effet (Blais, 2000, p. 163).

Dans le contexte de ce passage, l'euthanasie est l'approche clinique utilisant des moyens propres à soulager la souffrance. Elle constitue une intervention adéquate sur le plan de l'éthique, même si elle a pour effet d'abréger la vie.

Le Rapport du Comité sénatorial spécial sur l'euthanasie et l'aide au suicide contient un chapitre portant sur la terminologie pertinente. L'euthanasie y est définie ainsi: "Acte qui consiste à provoquer intentionnellement la mort d'autrui pour mettre fin à ses souffrances » (Sénat du Canada, 1995: 15). Cette définition diffère grandement de la précédente, puisque la mort y est un passage obligé dans le but de mettre un terme aux souffrances, plutôt qu'un effet non recherché de la thérapeutique employée. L'intention de procurer l'euthanasie ainsi définie se distingue de l'intention motivant un soulagement des souffrances au risque d'abréger la vie.

Considérons enfin une troisième définition apparaissant dans Le trésor de la langue française informatisé: «mort douce, de laquelle la souffrance est absente, soit naturellement, soit par l'effet d'une thérapeutique dans un sommeil provoqué » (Dendien, 2012). La mention du sommeil provoqué introduit un détail qui n'apparaît pas dans les autres définitions citées et qui semble s'apparenter à la notion de sédation terminale.

Il est impossible à des interlocuteurs qui discutent de l'euthanasie d'avancer dans leur discussion si l'un comprend l'euthanasie selon la première définition, un autre selon la seconde, et un autre encore selon la troisième. Il n'existe pas une bonne et de mauvaises définitions de l'euthanasie. Toutefois, l'objectif d'avancer dans la discussion rappelle la nécessité de préciser ce dont on parle. Conséquemment, toute discussion sur l'euthanasie doit débuter par l'adoption d'une définition.

À titre d'illustration, nous pourrions définir ainsi l'euthanasie: une action visant à provoquer le décès d'une personne atteinte d'une maladie incurable qui lui impose des souffrances physiques ou psychologiques intolérables, mise en œuvre à la demande de cette personne et pratiquée par un médecin ou sous sa supervision. Le Code criminel interdit l'euthanasie ainsi définie par ses dispositions sur l'homicide, particulièrement celles qui se rapportent au meurtre et au fait de conseiller le suicide ou d'y aider.

\section{L'ÉVITEMENT \\ DE LA CIRCONSTANCE LIMITE}

Malgré les progrès remarquables des soins palliatifs depuis une vingtaine d'années, il demeure des situations pour lesquelles les soins palliatifs sont moins efficaces qu'on le souhaiterait. Ces situations sont celles de personnes atteintes de souffrances physiques ou psychologiques intolérables pour elles, alors qu'elles anticipent «la perte de la vie corporelle, qui par nature fait horreur à la nature humaine » (Thomas d'Aquin, IIIa, q. 46, a. 6, Ad 1, 1986: 340). Les professionnels ou les proches de ces personnes peuvent leur offrir une solution comportant des changements à la médication, diverses interventions cliniques possiblement plus satisfaisantes, une aide spirituelle et une présence plus assidue. Cette première solution manifeste dévouement et imagination. Par contre, que faire si la personne concernée persiste à demander qu'on l'aide dans son projet de mourir?

Nous nous trouvons face à une circonstance limite lorsque la personne concernée affirme que les soins palliatifs ne la soulagent pas adéquatement de sa souffrance physique ou psychologique, et qu'elle demande qu'on l'aide à terminer ses jours. L'inévitabilité de ce type de situation nous place devant la nécessité de choisir entre accepter ou refuser d'aider la personne à mourir. Si notre choix est de refuser de l'aider à mourir, nous savons que nous ne répondons pas à ses attentes ou à l'expression de son autodétermination. L'évitement de la circonstance limite se produit lorsque la dispensation de soins palliatifs est perçue comme adéquate dans toutes les situations, incluant celles dans lesquelles la personne concernée continue à demander qu'on l'aide à mourir ${ }^{2}$. Nous devons reconnaître explicitement cette réalité que les soins palliatifs ne constituent pas nécessairement une réponse efficace à toutes les souffrances en fin de vie. 


\section{MAINTENIR OU NON L'INTERDICTION DE L'AIDE AU SUICIDE}

Voici quelques raisons fréquemment évoquées pour maintenir l'interdiction de l'aide au suicide:

- les demandes d'assistance à suicide ne manifestent pas toutes un désir réel ou définitif de mourir afin d'abréger les souffrances;

- la promotion de la vie implique le respect de son caractère sacré;

- l'amoindrissement du caractère sacré de la vie occasionne un préjudice moral au patient, aux médecins et à l'ensemble de la société;

- le phénomène appréhendé de la pente dangereuse pourrait prendre deux formes: une érosion progressive des mécanismes juridiques de protection de la vie et l'adoption de conduites de plus en plus permissives à l'égard des contraintes légales;

- la crainte des patients actuels et potentiels que leur mort soit provoquée sans qu'ils l'aient demandé;

- la difficulté d'opérer suivant des concepts clairs, notamment sur ce qu'est la phase terminale;

- chez certains médecins, la notion que le premier devoir de leur profession se trouve dans le mouvement de respecter la vie;

- l'appréhension que la valeur de la vie d'une personne puisse être établie à partir de son utilité sociale ou économique.

Voici certains motifs fréquemment évoqués pour modifier la loi de manière à permettre l'aide au suicide:

- la souffrance physique ou psychologique est parfois pire que la perspective de la mort qui apparaît comme une délivrance;

- la personne concernée est seule détentrice des droits associés à ses dimensions corporelle et psychique;

- suivant le principe d'autodétermination, la personne doit pouvoir exercer un contrôle sur sa vie et son mourir;

- une vie qui vaut d'être vécue doit présenter une qualité acceptable aux yeux de l'intéressé.

\section{LA RÈGLE DE DROIT}

\section{ET LES SITUATIONS PARTICULIÈRES}

Dans l'état actuel de notre droit, un acte qui provoque la mort d'autrui ou qui fournit une aide afin qu'une personne mette fin à sa vie constitue une infraction criminelle. D'aucuns estiment que la législation actuelle est opportune et devrait être maintenue. D'autres revendiquent des modifications considérables à la loi. Nonobstant mon point de vue particulier concernant la sagesse de la loi, que feraisje si une personne que j'aime, en proie à une souffrance terrible, me demandait avec détermination de l'aider à mourir ? Comment vivrais-je ensuite, considérant le poids moral de ma décision, quelle qu'elle soit? Que ferais-je si j'étais le médecin d'une personne très souffrante qui me demanderait d'abréger ses jours? Le point de vue du législateur et celui de la personne placée en situation présentent de profondes disparités ${ }^{3}$. Justifier sur le plan éthique la mise en œuvre d'une action particulière est différent de justifier, en vue du bien commun, une loi ou une bonne pratique professionnelle 4 . Une loi qui interdit l'aide au suicide peut être raisonnable, même si elle rend illégaux des actes qui, en eux-mêmes, sont moralement justifiés ${ }^{5}$. L'opération de la morale individuelle et l'élaboration des lois ne reposent pas sur les mêmes préceptes.

Shakespeare expose l'abîme qui sépare la sagesse et le détachement du bienportant de la passion subjuguant celui qui souffre:

Vois-tu, frère, les gens peuvent donner des conseils et parler de calme à une douleur qu'ils ne ressentent pas; mais, dès qu'ils l'éprouvent eux-mêmes, vite elle se change en passion, cette sagesse qui prétendait donner à la rage une médecine de préceptes, enchaîner la folie furieuse avec un fil de soie, charmer l'angoisse avec du vent et l'agonie avec des mots! Non! Non! C'est le métier de tout homme de parler de patience à ceux qui se tordent sous le poids de la souffrance; mais nul n'a la vertu ni le pouvoir d'être si moral, quand il endure lui-même la pareille. Donc ne me donne plus de conseils: ma douleur crie plus fort que les maximes (Shakespeare, 1959, p. 1319).

La loi s'exprime d'une façon générale afin de couvrir l'ensemble des situations. Elle ne peut toutefois pas prévoir toutes les circonstances possibles. Face à une situation aussi extrême que la fin de vie, la faculté de ressentir la souffrance de quelqu'un, l'empathie ${ }^{6}$, ne peut se réaliser qu'approximativement, puisque je ne suis pas l'autre. Mais cet effort pour pratiquer l'empathie permet d'être sensible à la souffrance d'autrui et engage à ne pas rejeter sommairement la possibilité que l'élimination de la souffrance commande des gestes sanctionnés par la loi.

\section{LE DOCUMENT DE TRAVAIL DE LA CRD}

L'avant-propos du document de travail de la CRD identifie les deux objectifs que poursuit le document. Il s'agit d'abord de considérer certains problèmes moraux et juridiques que posent la cessation de traitement et l'euthanasie, de percevoir comment ces problèmes affectent l'état du droit en vigueur, et d'examiner leurs effets dans une perspective de réforme législative. Deuxièmement, le document veut explorer certaines questions fondamentales de politique sociale pour encourager l'émergence d'un débat public. La CRD perçoit ce débat comme devant précéder une éventuelle réforme juridique. Dans cette optique, la loi intervient comme la manifestation juridique d'une orientation sociale générale.

\section{LA DÉFINITION DES PROBLÈMES}

Dans sa première partie, le document de travail de la CRD rappelle que la vie humaine constitue une valeur fondamentale des systèmes de droit modernes. Les lois interdisent l'homicide et elles punissent les actes qui constituent un danger ou une menace pour la vie d'autrui. Les dispositions du Code criminel canadien sur l'homicide considèrent la vie humaine dans une perspective quantitative et non qualitative. Il y a homicide si la personne touchée décède. Il n'y a pas d'homicide si la personne continue de vivre en étant atteinte d'un préjudice grave, comme l'incapacité de mener une vie relationnelle. Cette approche du droit reflète l'idée que la vie mérite une protection particulière, qu'elle est porteuse d'un caractère sacré.

\section{LE VITALISME ET LA QUALITÉ DE VIE}

Pour un certain courant de pensée, le vitalisme, la vie est une valeur suprême et tous les moyens doivent être employés pour la protéger, pour la prolonger et pour combattre la mort. Le vitalisme a exercé une influence sur la conduite médicale en incitant le médecin à repousser agressivement la maladie et la mort. Par ailleurs, les avancées scientifiques et technologiques permettent à la médecine de guérir des patients qui seraient autrefois décédés. Il s'agit là d'un incontestable progrès. D'un autre côté, ce progrès a compliqué les ques- 
tions humaines et juridiques touchant la mort. Des personnes que l'on a maintenues en vie se retrouvent parfois porteuses d'une qualité de vie peu enviable, par exemple celles dont l'existence sera végétative, sans espérance de retrouver une vie cognitive et relationnelle. Le constat de ces situations a engendré une réflexion concernant l'usage de la technologie médicale. Le critère d'utilisation basé sur la technique favorise l'intervention si elle est techniquement possible. Le critère d'utilisation basé sur des considérations humaines pour le patient favorise l'intervention si elle est humainement souhaitable. Cette distinction entre ce qui peut et ce qui doit être fait conduit au concept d'acharnement thérapeutique par lequel on désigne une attitude qui détourne la technologie de sa finalité réelle, qui est d'être placée au service de l'humain. Une technologie qui maintient en vie des personnes à tout prix ne réussit pas à prolonger la vie, mais à étirer le processus de la mort. En conséquence, il faut reconsidérer le caractère absolutiste de l'approche vitaliste.

Un nouveau courant de pensée est apparu dans les pays occidentaux. La vie demeure une valeur fondamentale, mais cette reconnaissance est tempérée par une préoccupation pour la qualité de vie. Ce changement est basé sur trois facteurs. Premièrement, une personne qui veut mourir dans la dignité peut rejeter l'utilisation abusive de la technologie médicale. Deuxièmement, le patient exige une participation directe dans le choix des soins qui lui seront prodigués. Le respect de cette autonomie du patient peut conduire à des décisions qui privilégient la qualité de vie, plutôt que sa durée. Troisièmement, le développement des soins palliatifs permet de cesser les interventions thérapeutiques et de dispenser des soins destinés à assurer un certain confort en phase terminale. L'avènement des soins palliatifs permet de sortir du choix entre un traitement inutile et l'absence totale de soins.

\section{LES PROBLÈMES JURIDIQUES TOUCHANT L'INTERRUPTION DE TRAITEMENT}

Trois difficultés se rattachent à l'interruption de traitement. Tout d'abord, notre société se trouve dans une période de transition où les attitudes concernant la vie, la mort et l'interruption de traitement sont en mutation, alors que les mentalités n'ont pas encore cessé d'évoluer. La protection de la vie humaine demeure importante, mais beaucoup de personnes souhaitent qu'on tienne davantage compte de l'autonomie personnelle et de la qualité de vie. Toutefois, la CRD considère que l'impossibilité d'identifier au moins deux cas iden- tiques rend difficile l'adoption de normes générales.

En second lieu, la relation entre la règle de droit et la pratique médicale présente des ambiguïtés. Une règle de droit du droit criminel a pour objet d'interdire une conduite jugée répréhensible, et elle joue un rôle répressif. Or, le document de la CRD souligne qu'aucun tribunal canadien n'a condamné un médecin pour avoir abrégé la vie d'un patient en phase terminale à l'aide de médicaments, pour avoir interrompu un traitement devenu inutile ou pour avoir refusé de prolonger l'agonie d'un patient. Par contre, cette absence de condamnation ne signifie pas que de tels événements ne se sont jamais produits. L'absence de condamnation judiciaire ne permet pas de considérer l'état actuel du droit comme satisfaisant. Plusieurs médecins souhaitent être guidés par un énoncé précis de leurs obligations légales, plutôt que de dépendre de la discrétion en matière de poursuite qu'exerce la Couronne. L'idéal serait que les médecins et les juristes puissent prédire comment la loi sera interprétée dans un cas de cessation de traitement.

La troisième difficulté concerne la possibilité que l'imprécision de la loi actuelle engendre une grande variété de pratiques dans les diverses régions du pays. Plutôt qu'être prévisibles et uniformes, les décisions des médecins seraient subjectives et fortement influencées par la personnalité de leur auteur.

Selon une certaine perception de l'état du droit, les médecins ont peu à craindre par rapport au droit criminel. Par contre, il s'agirait de quelques événements pour que des médecins deviennent l'objet du cas type en droit criminel. Les questions relatives à la cessation ou la non-initiation d'un traitement sont trop importantes pour être abandonnées à une telle incertitude.

\section{DEUX CAS DE FIGURE}

Le document de la CRD campe sa réflexion dans deux cas de figure: le malade en phase terminale et le nouveauné souffrant de malformations graves. Le malade en phase terminale est celui pour qui la dispensation de soins thérapeutiques ne peut plus assurer une guérison ou un certain contrôle sur la maladie. L'intérêt du patient consiste alors dans des soins destinés à soulager ses souffrances physiques et morales, pour lui procurer une fin de vie dans la dignité et la lucidité. Cette approche de la phase terminale produit deux problèmes. Premièrement, la théorie et la tradition juridiques veulent que l'interruption d'une mesure destinée à sauver la vie d'une personne puisse fonder une responsabilité civile et criminelle. La crainte de poursuites civiles et criminelles est susceptible de pousser un médecin à maintenir un traitement devenu inutile ou de le cesser tardivement. Au contraire, il faudrait que la loi établisse l'idée du droit de mourir dans la dignité, sans souffrir des interventions qui sont de l'acharnement thérapeutique. Deuxièmement, l'administration de médicaments afin d'éliminer ou de réduire la souffrance peut avoir comme effet secondaire d'abréger la vie. Certains médecins craignent d'être l'objet de difficultés pénales, civiles ou disciplinaires pour avoir dispensé des soins palliatifs qui auraient abrégé la vie du patient. Il faut modifier la loi de manière à éliminer cette menace virtuelle pesant sur le médecin qui prodigue des soins adéquats en phase terminale.

Le nouveau-né souffrant de malformations graves se trouve lui aussi au centre de questions juridiques considérables. Depuis le milieu des années 1950, la médecine a développé des instruments propres à sauver des enfants qui seraient décédés dans le contexte antérieur. Par contre, ces progrès ont engendré de nouveaux problèmes puisque certains de ces enfants, porteurs d'anomalies graves, seront confiés à des institutions ou rejoindront leur famille en lui imposant un énorme fardeau financier, affectif et psychologique. Une seconde considération est que d'autres personnes que le nouveau-né décident pour lui de sa vie ou de sa mort. Il est évidemment impossible de savoir ce que lui-même aurait souhaité. Une troisième considération vient affecter la perception de celui qui décide pour l'enfant: le nouveau-né est un être humain dont la vie commence à peine, et pour qui la mort est déjà une éventualité.

Pour le médecin, la situation du nouveau-né présente des analogies avec celle du patient en phase terminale. Dans les deux cas, si le médecin entreprend un traitement, la loi lui impose-t-elle de poursuivre ce traitement, même s'il devient inutile? Dans le cas du nouveauné, le médecin doit-il agir avec l'accord des parents? Les parents sont-ils les seules personnes à pouvoir prendre la décision?

\section{LES RÉPONSES DU DROIT CRIMINEL} EN 1982

Face à ces questions, le droit en vigueur au moment de la publication du document de la CRD offre certaines réponses. Nous allons parcourir le contenu pertinent $\mathrm{du}$ Code criminel canadien. Suivant l'article 197, certains individus ont l'obligation de fournir les choses nécessaires à la vie aux personnes qui dépendent d'eux. La jurisprudence a interprété cette disposition comme s'appliquant à un membre du personnel médical qui néglige ou refuse 
de fournir des soins médicaux à une personne, si cette personne ne se trouve pas en mesure de prendre soin d'elle-même. Selon cette interprétation, un médecin qui négligerait de dispenser un traitement à un patient inconscient pourrait être passible d'une poursuite criminelle.

L'article 198 contraint celui qui entreprend un traitement médical ou chirurgical pouvant mettre la vie en danger, à employer une connaissance, une habileté et des soins raisonnables. L'utilisation du mot "raisonnable» fait référence à ce qui peut être considéré comme médicalement raisonnable dans chaque cas. Un tribunal pourrait estimer raisonnable qu'un médecin ne tente pas de réanimer une personne en coma dépassé. Par contre, le médecin serait-il exonéré si, pour des motifs reliés à la qualité de vie du nouveau-né, il n'avait pas procédé à une intervention chirurgicale bénigne qui l'aurait maintenu en vie?

L'article 199 contraint celui qui entreprend un acte à le continuer si l'omission de le faire comporte un danger pour la vie. En matière de traitement médical, cet article a une importance, puisque son dispositif pourrait s'appliquer à l'interruption de traitement. En effet, la conduite du médecin qui pratique une assistance respiratoire est-elle visée lorsqu'il interrompt le traitement par l'arrêt du respirateur? L'article 199 semble énoncer que le médecin qui a établi un traitement ne peut pas le cesser si cette cessation représente un risque pour la vie du patient. Cette interprétation de l'article 199 contraindrait les médecins à pratiquer l'acharnement thérapeutique. Elle aurait vraisemblablement pour effet de faire hésiter les médecins à entreprendre un traitement qu'ils ne pourraient cesser. Une règle de cette nature serait dommageable pour la pratique médicale.

Cependant, l'interprétation de l'article 199 doit tenir compte de l'article 45 et des dispositions portant sur la négligence criminelle. L'article 45 protège de la responsabilité criminelle celui qui a pratiqué une intervention chirurgicale pour le bien d'une personne, alors qu'il était raisonnable de la pratiquer compte tenu de l'état de santé du patient. Le standard de conduite que retient l'article 45 est le caractère raisonnable de l'acte dans les circonstances. Les dispositions concernant la négligence criminelle ne pénalisent pas toute interruption de traitement qui met la vie en danger, mais celle qui manifeste une insouciance déréglée ou téméraire.

Les articles 202 à 223 portent sur la négligence criminelle et les différentes sortes d'homicide. Quant au meurtre, la loi ne tient aucun compte de la motivation de son auteur. Que le meurtre soit motivé par la vengeance, le lucre ou la compassion ne fait pas de différence. Ce qui compte, c'est que son auteur avait l'intention de provoquer la mort.

L'article 14 déclare que le consentement de la personne à ce qu'on lui donne la mort n'a pas d'influence sur la responsabilité pénale de l'auteur. Conséquemment, celui qui donne la mort à une personne qui le lui demande afin d'abréger ses souffrances est coupable de meurtre.

Enfin, l'article 224 établit une infraction pour celui qui conseille à une personne de se suicider ou l'aide à le faire. Cette infraction est maintenue, même si la tentative de suicide n'est plus un acte criminel.

\section{L'APPLICATION DES TEXTES}

Ces dispositions du Code criminel ont donné lieu à une jurisprudence considérable. Cependant, en 1982, la jurisprudence appliquant ces dispositions au contexte médical est rarissime. Il existe

\section{AU CANADA,}

IL EXISTE UNE INCERTITUDE

FACE À LA LOI, PUISQUE

LES DISPOSITIONS PERTINENTES

DU CODE CRIMINEL SONT

D'APPLICATION GÉNÉRALE,

QU'ELLES N'ONT PAS ÉTÉ

CONÇUES PRÉCISÉMENT

POUR LE CONTEXTE MÉDICAL.

DE PLUS, LA RARETÉ

DE LA JURISPRUDENCE

NOUS PRIVE DE CONNAÎTRE

L'INTERPRÉTATION QUE LEUR

DONNERAIENT LES TRIBUNAUX.

donc un décalage entre la rigueur de ces dispositions et leur application pratique dans le contexte médical. Ce décalage s'explique d'abord par le fait que le standard de négligence requis pour l'existence d'une responsabilité pénale est élevé. Très peu de médecins canadiens adoptent ce type de comportement. Deuxièmement, établir le lien de causalité entre l'acte posé et le résultat est difficile. Troisièmement, notre système de justice pénale effectue un processus de filtrage des poursuites suivant la discrétion dont dispose le bureau du procureur général. La CRD observe qu'en dehors du contexte médical, les poursuites criminelles pour meurtre par compassion sont très rares dans l'histoire du Canada. La rareté de la jurisprudence fait que nous ne savons pas précisément comment appliquer les dispositions du Code criminel au contexte médical et au meurtre par compassion.

Le document contient un bref survol de l'état du droit dans les pays suivants: Grande-Bretagne, États-Unis, Allemagne, Suisse, Norvège et Uruguay. Au Canada, il existe une incertitude face à la loi, puisque les dispositions pertinentes du Code criminel sont d'application générale, qu'elles n'ont pas été conçues précisément pour le contexte médical. De plus, la rareté de la jurisprudence nous prive de connaître l'interprétation que leur donneraient les tribunaux. Au moment de la rédaction du document de la CRD, tous les systèmes juridiques avaient refusé d'admettre l'euthanasie active et volontaire. Par contre, dans tous les pays, nous relevons une contradiction entre la sévérité de la loi et la pratique judiciaire: les auteurs de certains actes sont rarement accusés, et s'ils sont reconnus coupables, leurs peines sont légères.

\section{LA NÉCESSITÉ ET LES OBJECTIFS DE LA RÉFORME}

Certains peuvent estimer qu'aucune réforme de la loi n'est requise, puisque les dispositions pertinentes du Code criminel ont déjà fait l'objet de certaines interprétations, et qu'elles sont rarement appliquées dans le contexte médical. Par contre, la CRD recommande une réforme qui réaménagerait et compléterait des dispositions en vigueur. Premièrement, le caractère général des dispositions en vigueur a pour avantage de permettre leur application durant une longue période de temps. Cependant, ces dispositions suscitent des incertitudes quant aux situations nouvelles. Deuxièmement, en plus de son rôle répressif, la loi exerce un rôle préventif. Or, la loi n'offre pas d'indications utiles concernant les nouvelles pratiques médicales et hospitalières, notamment celles qui sont mises en œuvre à l'égard des mourants et des nouveau-nés souffrant de malformations graves.

Les discussions publiques sur une réforme législative peuvent mener à un niveau plus ou moins élevé de consensus social. Par contre, il serait étonnant qu'une réforme législative fasse l'unanimité. Enfin, la réforme législative ne doit pas être perçue comme la finalité à atteindre, 
mais plutôt comme le début d'une réforme plus large, à la fois sociale et institutionnelle.

\section{LES IMPÉRATIFS DE LA RÉFORME}

Le document de la CRD énonce ses préoccupations à l'égard d'une réforme $\mathrm{du}$ droit criminel sous la forme de trois questions:

(1) Le législateur doit-il, d'une façon ou d'une autre, légaliser ou du moins décriminaliser certaines formes d'euthanasie active, comme le meurtre par compassion? (2) Le législateur doit-il décriminaliser l'aide au suicide en abrogeant l'article 224 du Code criminel? (3) Le législateur doit-il intervenir dans le cadre des articles 14, 45, 198 et 199, pour préciser les limites de la légalité du refus et de la cessation du traitement médical (Commission de réforme du droit du Canada, 1982, p. 36-37)?

Les réponses apportées à ces questions doivent être guidées par certains principes ou règles fondamentales. Sur le plan de la nature de la réforme, celle-ci doit être consciente des limites du droit criminel, les solutions proposées doivent être suffisamment souples pour s'appliquer aux divers contextes, et les solutions doivent s'insérer harmonieusement dans le contexte social et juridique actuel.

Sur le plan du contenu, la CRD identifie six principes. Le premier principe est le refus de fonder une réforme sur la reconnaissance de deux catégories d'êtres: les personnes humaines et les non-personnes. La CRD affirme clairement que le statut d'humain est applicable à tous ceux qui sont nés de parents humains. Deuxièmement, la CRD ne fait pas sienne la distinction connue entre mesures ordinaires et mesures extraordinaires. Cette distinction est trop ambiguë pour qualifier avec précision l'intensité de l'obligation du médecin à l'égard de son patient. Troisièmement, la préservation de la vie humaine est une valeur fondamentale dans notre société. Une réforme du droit ayant un impact sur la vie humaine doit admettre une présomption en faveur de la vie, sans toutefois requérir l'acharnement thérapeutique: "si un traitement peut être raisonnablement appliqué pour préserver la vie ou la santé d'une personne, on doit présumer que la volonté de cette personne, si elle avait pu la manifester, eût été de recevoir le traitement et non de le refuser» (Commission de réforme du droit du Canada, 1982: 42). De cette façon, le fardeau de la preuve incombe à celui qui, dans un cas réel, ne favorise pas le prolongement ou le maintien de la vie. Le quatrième principe reconnaît l'autonomie et l'autodétermination de la personne, puisque chacun est maître de sa destinée. En conséquence, chaque personne est libre de refuser un traitement ou de demander sa cessation. Cinquièmement, la vie humaine ne comporte pas seulement un aspect quantitatif, mais aussi un aspect qualitatif. Des considérations portant sur la qualité de vie peuvent être légitimes dans certaines décisions, notamment lorsque le patient refuse des traitements. Il revient au patient d'exercer son autonomie en définissant ses priorités et ses exigences personnelles quant à sa qualité de vie. Enfin, les personnes dites «incapables» ou plus faibles doivent bénéficier d'une protection particulière parce que leurs droits peuvent être plus facilement violés ou ignorés. Lorsque la personne concernée ne peut plus prendre les décisions concernant sa santé ou sa vie, le consentement substitué est la solution applicable. Par contre, nul ne peut être certain que la personne ellemême prendrait les mêmes décisions.

\section{LA RÉFORME - LÉGALISATION DE L'EUTHANASIE}

Lorsqu'il entreprend de répondre à la première question, le document précise ce qu'il entend par euthanasie: «l'acte positif de causer la mort de quelqu'un pour des raisons humanitaires » (Commission de réforme du droit du Canada, 1982: 50). Les tenants de la légalisation de l'euthanasie permet implicitement au patient en phase terminale de s'enlever la vie.

La CRD estime que la légalisation de l'euthanasie n'est pas acceptable, en raison de la disproportion que représentent ses risques par rapport aux profits attendus. La position de la CRD repose sur trois arguments. L'argument principal est celui des abus possibles. L'euthanasie pourrait servir à éliminer ceux qui représentent un fardeau pour les autres ou pour la société. Il se peut aussi que le consentement à l'euthanasie ne soit pas toujours un acte libre et volontaire, en dépit des précautions que contiendrait la loi. Deuxièmement, les procédures entourant la pratique de l'euthanasie introduiront une bureaucratisation du processus de décision. Or, les délais sont un des maux que les tenants de l'euthanasie veulent éviter. Troisièmement, une modification législative doit comporter une proportion entre les maux que l'on veut éviter et les risques nouveaux que crée la modification. Cette proportion est inacceptable dans le cas de l'euthanasie.

\section{LA RÉFORME - DÉCRIMINALISATION DU MEURTRE PAR COMPASSION}

L'examen de l'hypothèse de la décriminalisation du meurtre par compassion invite à examiner trois possibilités: conserver intégralement le régime actuel, qui ne tient pas compte du motif ayant poussé à l'homicide; créer une catégorie d'homicide qui tienne compte des cas où le motif est excusable;

LES TENANTS DE LA LÉGALISATION DE L'EUTHANASIE SOULIGNENT CE QU'ILS ESTIMENT ÊTRE L'ILLOGISME DE LA LOI. LE PATIENT DÉTIENT LE DROIT DE REFUSER UN TRAITEMENT ET CELUI DE REQUÉRIR SON INTERRUPTION, AFIN DE NE PAS PROLONGER SON AGONIE. LE FONDEMENT DE CE DROIT EST QUE LE PATIENT EST MAÎTRE DE SA PERSONNE. SI L'AUTONOMIE DÉCISIONNELLE PERMET UNE OMISSION OU UNE ABSENCE D'ACTION, POURQUOI NE PERMET-ELLE PAS DE POSER UN ACTE POSITIF ?

soulignent ce qu'ils estiment être l'illogisme de la loi. Le patient détient le droit de refuser un traitement et celui de requérir son interruption, afin de ne pas prolonger son agonie. Le fondement de ce droit est que le patient est maître de sa personne. Si l'autonomie décisionnelle permet une omission ou une absence d'action, pourquoi ne permet-elle pas de poser un acte positif? De plus, puisque depuis 1972, la tentative de suicide n'est plus un acte criminel, le droit maintenir l'infraction actuelle, mais tenir compte du motif dans le choix de la sentence. La qualification du motif ayant poussé à l'homicide est un problème considérable, puisque des meurtres crapuleux pourraient être commis sous le couvert de l'homicide par compassion. La CRD perçoit une solution possible dans la reconnaissance que l'acte posé continue d'être un meurtre, mais que le motif de la compassion ait un impact majeur sur la sentence. Cette position de la CRD n'est pas définitive. 


\section{LA RÉFORME - DÉCRIMINALISATION DE L'AIDE AU SUICIDE}

La seconde question demande si le législateur devrait décriminaliser l'aide au suicide. Comme nous l'avons déjà mentionné, la tentative de suicide n'est plus un acte criminel. Dans certains cas, la personne atteinte d'une maladie dégénérative peut souhaiter vivre encore jusqu'à un moment où elle préférera mourir. Il se peut qu'à ce moment, elle ne dispose plus de la capacité physique de procéder. Pourquoi interdire qu'une personne l'assiste dans son projet? La CRD reconnaît qu'il semble, «à première vue, fort incongru de criminaliser la participation à un acte qui n'est plus lui-même un acte criminel » (Commission de réforme du droit du Canada, 1982: 60). L'interdiction d'aider une personne à mourir ne vise pas que les cas de phase terminale. Cette interdiction est beaucoup plus vaste. Elle protège notamment la personne dépressive que quelqu'un pourrait encourager à mourir, non pas pour des motifs humanitaires, mais pour des avantages financiers. Décriminaliser entièrement l'aide au suicide n'est probablement pas une politique souhaitable sur un plan général. Par contre, serait-ce une initiative avantageuse s'agissant de personnes en phase terminale? Comme pour l'homicide par compassion, la motivation de la personne qui intervient est, en principe, de nature humanitaire. Toutefois, comment nous assurer de l'intention réelle de l'auteur de l'acte? Il se peut que, pour des motifs peu nobles, quelqu'un commette l'homicide d'une personne en phase terminale, et réussisse à camoufler son acte en une aide au suicide. Par contre, la CRD reconnaît la possibilité qu'une personne en aide une autre à mourir, alors que son intention est exclusivement humanitaire. La CRD recommande donc un amendement à l'article 224 du Code criminel précisant qu'une poursuite pour aide au suicide ne peut être entreprise sans l'autorisation du procureur général lui-même.

\section{LA RÉFORME - INTERRUPTION DE TRAITEMENT ET RENONCIATION AU TRAITEMENT}

La troisième question demande si le législateur devrait intervenir pour préciser les limites de la légalité du refus et de la cessation du traitement médical. La CRD note qu'il existe une distance considérable entre la pratique médicale et la conduite que pourrait autoriser une interprétation restrictive des dispositions du Code crimi$n e l$. Une personne capable de décider doit pouvoir exercer le droit de refuser un traitement et celui d'en demander la cessation. Toutefois, le droit criminel semble impo- ser la continuation du traitement entrepris si sa cessation représente une menace pour la vie. Cette interprétation du droit criminel paraît encourager l'acharnement thérapeutique. Or, il existe une différence entre l'acte qui provoque la mort et le fait de cesser un traitement lorsque le patient le demande. Le document approche cette question d'abord pour la personne capable de décider, puis, pour la personne incapable. Rappelant le concept de la mort dans la dignité, la CRD propose de reconnaître aussi clairement que possible, pour toute personne capable, le droit absolu de refuser ou d'interrompre un traitement médical qui lui est destiné.

Le cas de la personne incapable est plus complexe. Une personne incapable est «tout être humain qui, par la suite d'un défaut d'âge, d'un état d'inconscience temporaire ou permanent, ou d'un handicap, est dans l'impossibilité de manifester sa volonté et de prendre une décision éclairée et perd ainsi sa faculté de choisir » (Commission de réforme du droit du Canada, 1982: 65). La CRD énonce que l'incapacité d'une personne ne doit pas servir à nier ses droits fondamentaux. La personne incapable doit bénéficier d'une protection accrue de la loi. Les mécanismes de protection comme la curatelle existent pour que les décisions prises au nom de la personne représentée soient raisonnables, aussi à l'abri que possible de l'arbitraire. Pour le médecin, l'objectif poursuivi ne devrait pas être le maintien en vie, mais le bien du patient. Une façon de se représenter le bien du patient est de se rappeler les volontés qu'il aurait pu exprimer sur le sujet. Par contre, certaines personnes n'ont jamais pu s'exprimer sur de telles questions: c'est le cas du nouveau-né et de la personne atteinte d'une déficience intellectuelle majeure. Pour ces personnes, différents modes décisionnels sont possibles. La décision pourrait être prise par le médecin, par un tribunal ou par les proches, dont le curateur. Après avoir envisagé les avantages et les inconvénients de chaque mode, la CRD conclut que le moins mauvais semble être celui qui confie la décision au médecin.

\section{LES RECOMMANDATIONS DE RÉFORME}

En réponse aux trois questions qu'elle a posées, la CRD formule certaines recommandations. Sur l'euthanasie active, la CRD recommande que les prohibitions en vigueur concernant l'homicide soient maintenues. Il faut aussi maintenir l'interdiction du meurtre par compassion. Cependant, le motif compassionnel pourrait intervenir pour une réduction possible de la sentence. Sur l'aide au suicide, la CRD conclut «qu'une décriminalisation complète de l'aide ou de l'incitation au suicide serait inopportune et dangereuse dans le contexte actuel » (Commission de réforme du droit du Canada, 1982: 78-79). Toutefois, la CRD propose de modifier l'article 224 par l'addition d'un second paragraphe précisant qu'une poursuite en vertu de cet article doit avoir été autorisée par le procureur général lui-même. Sur l'interruption de traitement et la renonciation au traitement, la CRD suggère l'addition au Code criminel d'un texte établissant que le médecin n'a pas l'obligation d'administrer un traitement lorsque le patient le refuse ou lorsque ce traitement est médicalement inutile et n'est pas dans le meilleur intérêt du patient, sauf si celui-ci a clairement manifesté sa volonté du contraire. La CRD propose aussi l'addition d'un texte protégeant de la responsabilité criminelle le médecin qui dispense des soins palliatifs ou des mesures destinées à éliminer ou à atténuer les souffrances, alors que ces soins ou ces mesures sont susceptibles de raccourcir l'espérance de vie du patient.

\section{LE PROJET DE LOI C-384}

Le 13 mai 2009, madame Francine Lalonde, députée de La Pointe-de-l'Île, a présenté à la Chambre des communes son projet de loi C-384. Le dispositif de ce projet de loi autorise l'intervention d'un médecin pour aider une personne âgée d'au moins 18 ans à «mourir dignement». Le sommaire du projet de loi expose qu'il s'agit de modifier le Code criminel afin de permettre à un médecin, suivant certaines conditions, "d'aider une personne qui éprouve des douleurs physiques ou mentales aiguës sans perspective de soulagement ou qui est atteinte d'une maladie en phase terminale à mourir dignement quand elle y consent de façon libre et éclairée».

Le texte du projet de loi C-384 ne présente évidemment pas la réflexion qui se trouve derrière son dispositif. Par contre, la consultation de la transcription des débats à la Chambre des communes nous permet de mieux faire ressortir les intentions à l'origine du projet de loi. Nous espérons réussir à ne pas faire dire plus ni autre chose au projet de loi que ce qui était entendu par ses rédacteurs.

Lors d'un débat à la Chambre des communes le 2 octobre 2009, madame Lalonde a identifié précisément l'objectif poursuivi: «le droit d'avoir une fin de vie conforme aux valeurs de dignité et de liberté [...] En fait, c'est pour que les personnes aient le choix, le droit de choisir qui existe dans d'autres pays» (Lalonde, 2009: 1330). Madame Lalonde décrit ainsi la démarche concrète que la personne qui souhaite 
«mourir dignement» devrait effectuer en vertu du projet de loi C-384: «Cette personne doit avoir remis à un médecin deux demandes écrites à plus de 10 jours d'intervalle indiquant expressément - c'est dans le texte - son consentement libre et éclairé à opter pour la mort» (Lalonde, 2009: 1330). Lors d'un autre débat, tenu le 20 avril 2010, madame Lalonde exprime très explicitement que son projet de loi entend protéger les personnes vulnérables en affirmant leur droit de choisir : "Il faut avoir le droit de choisir. Je le dis pour les personnes vulnérables. Ce sont elles qui ont le plus besoin d'une loi parce que seule cette loi leur permettra d'être les personnes qui choisiront» (Lalonde, 2010, 1825). En fait, la consultation de la transcription des débats à la Chambre des communes révèle clairement que donner le droit de choisir aux personnes souffrantes constitue la motivation essentielle du projet de loi.

\section{L'AIDE D'UN MÉDECIN}

Seul un médecin peut aider une personne à «mourir dignement». Le projet de loi exclut la possibilité qu'un proche ou une autre personne fournisse cette aide. Nous pouvons raisonnablement supposer que le recours au médecin repose sur la présomption que celui-ci détient l'expertise et les techniques requises afin de procurer une mort dans la dignité. Précisons que rien, dans le projet de loi, ne contraint un médecin à fournir cette aide.

\section{DEUX TYPES DE SITUATIONS}

Un médecin peut aider une personne à «mourir dignement » dans deux types de situations. Dans le premier type, la personne "continue, après avoir essayé ou expressément refusé les traitements appropriés et disponibles, d'éprouver des douleurs physiques ou mentales aiguës sans perspective de soulagement». Cette ouverture existe, que la personne ait essayé ou refusé les traitements appropriés et disponibles.

Dans le second type de situations visées, la personne peut obtenir de mourir dignement si elle est atteinte d'une maladie en phase terminale. Le projet de loi ne précise pas ce qu'est la phase terminale. Par contre, l'hypothèse de la phase terminale correspond au type de contexte habituellement évoqué lors des débats sur la mort dans la dignité.

\section{DEMANDES ÉCRITES}

À l'égard de tous les cas, la personne doit avoir "remis à un médecin, alors qu'elle était apparemment lucide, deux demandes écrites à plus de dix jours d'intervalle indiquant expressément son consentement libre et éclairé à opter pour la mort». À quel état l'expression «apparemment lucide» fait-elle référence ? Cette terminologie diffère de celle qu'applique le droit civil québécois en matière de santé, soit la notion d'aptitude. Le projet de loi ne définit pas la lucidité apparente. Toutefois, il faut savoir que, dans une discussion approfondie sur la mise en œuvre de ce projet de loi, la signification de l'expression « apparemment lucide» serait cruciale.

\section{DÉSIGNATION D'UNE PERSONNE}

La personne doit aussi avoir «désigné, par un écrit fait avec son consentement libre et éclairé et devant deux témoins qui n'ont pas d'intérêt personnel dans sa mort, une autre personne qui agira en son nom auprès de tout médecin lorsqu'elle ne sera apparemment pas lucide». Ce procédé permet à une personne d'anticiper sa perte de lucidité et de prévoir son «mourir dignement» à la demande de la personne qu'elle a désignée. La personne concernée exerce en quelque sorte son autonomie au-delà de sa perte de lucidité. La désignation d'une autre personne semble particulièrement pertinente en cas de maladie neurodégénérative. Sans la faculté de désigner une personne pour agir en son nom lorsqu'elle ne sera plus lucide, la personne concernée se trouverait devant une alternative affligeante: soit terminer sa vie à un moment où celle-ci présente encore un certain intérêt, soit continuer à vivre après la perte de sa lucidité dans une condition diminuée qu'elle a anticipée et redoutée. Le document de la CRD aborde ce genre de situation lorsqu'il expose le point de vue des personnes favorables à la décriminalisation de l'aide au suicide: «Si elle est incapable de poser le geste elle-même (par exemple parce qu'elle est paralysée, ou ne peut avoir accès aux moyens nécessaires à la réussite de son entreprise), il est illogique, argumentet-on, de traiter comme un criminel celui qui l'aide à réaliser son plan (Commission de réforme du droit du Canada, 1982: 60). La possibilité de «mourir dignement» à la demande de la personne désignée se trouve en harmonie avec la préoccupation de madame Lalonde pour les personnes vulnérables. En effet, une personne en fin de vie, très affaiblie physiquement, et incapable de se donner elle-même la mort fait assurément partie des personnes vulnérables.

\section{RÉVOCATION DES DEMANDES ET CONFIRMATION DU DIAGNOSTIC}

La personne peut, à tout moment, révoquer ses deux demandes écrites. Madame Lalonde insiste sur cette liberté qu'a la personne de changer d'idée: «La personne est libre de changer d'idée. Le médecin doit lui rappeler cela constamment» (Lalonde, 2009, 1330). Toutefois, cette obligation qu'aurait le médecin de rappeler constamment l'existence de la possibilité de changer d'idée n'apparaît pas dans le projet de loi. Le médecin doit avoir « reçu une confirmation écrite du diagnostic d'un autre médecin» et n'avoir «aucun motif raisonnable de croire que les demandes écrites» de la personne «ont été faites sous la contrainte ou lorsque la personne n'était pas lucide». Le médecin doit aussi avoir «informé la personne des conséquences de ses demandes et des autres possibilités qui s'offrent à elle».

\section{« MOURIR DIGNEMENT » : UNE AIDE AU SUICIDE}

Par rapport aux notions utilisées dans le document de la CRD, le projet de loi C-384 se situe-t-il dans l'espace de l'euthanasie ou dans celui de l'aide au suicide? Rappelons que, pour la CRD, l'euthanasie est «l'acte positif de causer la mort de quelqu'un pour des raisons humanitaires». Ainsi définie, l'euthanasie peut être pratiquée sur une personne apte qui la demande, comme sur une personne inapte qui n'y a pas consenti. Or, le projet de loi C-384 ne propose pas quelque chose d'aussi général que d'autoriser l'acte de causer la mort pour des raisons humanitaires. Le caractère essentiel de la volonté qu'exprime la personne concernée d'obtenir l'aide d'un médecin fait de cette possibilité une forme d'aide au suicide.

\section{UNE SIMILITUDE FONDAMENTALE ET DES PARADIGMES DIFFÉRENTS}

Le document de la CRD et le projet de loi C-384 comportent une similitude fondamentale et des paradigmes différents. Le document de la CRD reconnaît le caractère sacré de la vie, mais cette reconnaissance doit trouver une limite dans une préoccupation pour la qualité de vie. Le principe du caractère sacré de la vie ne doit pas être perçu comme requérant l'acharnement thérapeutique. De plus, à titre d'agent détenteur d'autonomie, la personne a le droit de choisir les soins qui lui seront prodigués, et de privilégier la qualité de vie, plutôt que la quantité.

Le document de la CRD valorise considérablement la conjonction entre l'exercice de l'autonomie personnelle et l'appréciation de la qualité de vie. Pour la $\mathrm{CRD}$, la personne concernée procède à une appréciation de sa qualité de vie et décide des soins qu'elle recevra ou non. Le projet de loi C-384 est fondé sur un mouvement similaire: la personne qui exprime sa volonté de «mourir dignement »a apprécié sa qualité de vie présente ou éventuelle, et elle exerce son autonomie en deman- 
dant à mourir avec l'aide d'un médecin. La similitude, la relation, la caractéristique commune qui unit le document de la CRD et le projet de loi C-384 se trouve dans la conjonction entre l'exercice de l'autonomie personnelle et l'appréciation de la qualité de vie. Le document de la CRD applique l'exercice de l'autonomie au refus et à la cessation de traitement dans une opposition à l'acharnement thérapeutique, alors que le projet de loi C-384 étend les possibilités de l'autonomie au choix de «mourir dignement». Dans les deux documents, l'approche privilégiée est soutenue et renforcée par une valorisation de l'autonomie personnelle.

Il existe un aspect intéressant à relever concernant l'exercice de l'autonomie lorsque la personne est devenue incapable. Le projet de loi prévoit que la personne concernée désigne une autre personne pour la représenter «lorsqu'elle ne sera apparemment pas lucide». L'objectif de cette mesure semble

LE DOCUMENT DE LA CRD ET LE PROJET DE LOI C-384 COMPORTENT UNE SIMILITUDE FONDAMENTALE ET DES PARADIGMES DIFFÉRENTS. LE DOCUMENT DE LA CRD RECONNAÎT LE CARACTÈRE SACRÉ DE LA VIE, MAIS CETTE RECONNAISSANCE DOIT TROUVER UNE LIMITE

\section{DANS UNE PRÉOCCUPATION}

\section{POUR LA QUALITÉ DE VIE.}

être de s'assurer que les volontés de la personne seront respectées au-delà de sa perte de lucidité. Le document de la CRD exprime la même préoccupation concernant la protection des droits de la personne incapable: "L'incapacité doit continuer à être une mesure de protection; elle ne doit pas servir à retirer des droits à cette personne, ni surtout à aggraver sa situation face à la mort » (Commission de réforme du droit du Canada, 1982: 66). Une aggravation de la situation de la personne face à la mort serait, par exemple, de
LA SIMILITUDE, LA RELATION,

LA CARACTÉRISTIQUE COMMUNE

QUI UNIT LE DOCUMENT

DE LA CRD ET LE PROJET

DE LOI C-384 SE TROUVE

DANS LA CONJONCTION ENTRE

L'EXERCICE DE L'AUTONOMIE

PERSONNELLE ET L'APPRÉCIATION

DE LA QUALITÉ DE VIE.

lui imposer ce qu'elle considérerait comme de l'acharnement thérapeutique.

Le projet de loi C-384 place le droit de choisir, l'exercice de l'autonomie personnelle au sommet des valeurs, et en fait son paradigme. Par paradigme, nous entendons un modèle ou une représentation servant de référence. Suivant le paradigme de l'exercice de l'autonomie personnelle, la personne peut légitimement demander à «mourir dignement». Le document de la CRD attribue lui aussi une grande importance à l'exercice de l'autonomie personnelle, mais il estime que la décriminalisation de l'aide au suicide n'est pas acceptable en raison des dangers d'abus qu'elle comporte. Nous constatons que le paradigme de la CRD est plutôt mixte et constitue un arbitrage entre l'exercice de l'autonomie personnelle et les précautions à maintenir pour éviter que ne se réalisent les abus anticipés. Il tombe sous le sens que la pratique de tels abus porterait offense au caractère sacré de la vie.

L'expérience maintenant acquise dans les juridictions qui ont adopté une législation sur le «mourir dignement» permet peut-être de répondre aux craintes qu'a exprimées la CRD. Il s'agirait alors d'évaluer si l'ensemble de la procédure et des garde-fous qu'instaure le projet de loi C-384 est suffisant pour répondre à ces craintes.

\section{DES ASPECTS À EXPLORER}

Le document de la CRD formule des propositions concernant le refus et la cessation de traitement. L'évolution du droit à cet égard mérite notre attention, puisque les constats de la CRD à ce sujet sont dépassés. De plus, deux aspects du projet de loi sont de nature à produire des débats inévitables, parce que cruciaux: le consentement à sa propre mort, et les douleurs aiguës comme motif pour demander à «mourir dignement».

\section{LE REFUS ET LA CESSATION DE TRAITEMENT}

Depuis la parution du document de travail de la CRD en 1982, les débats concernant le contrôle à exercer sur notre mort nous ont conduits de la cessation de traitement à l'aide au suicide. En raison des développements jurisprudentiels survenus en 1992, le débat sur l'aide médicale au suicide concerne essentiellement les patients non maintenus en vie par des appareils et des interventions qui leur fournissent un traitement vital. Un traitement vital est celui qui maintient la personne en vie. Sans ce traitement, la personne décéderait à plus ou moins court terme.

Le droit du patient de refuser un traitement vital ou d'en exiger la cessation semble ne plus poser de difficulté de principe, ni juridique ${ }^{7}$ ni éthique. Lorsqu'il cesse un traitement vital, le médecin agit pour respecter la volonté du patient, et conformément au rôle professionnel que lui ont confié la société et la loi.

Le Barreau du Québec a produit en 2010 un mémoire intitulé Pour des soins de fin de vie respectueux des personnes. Une section de ce mémoire porte sur le droit du patient à l'interruption ou à la cessation de traitement et rappelle deux décisions judiciaires déterminantes survenues en 1992:

Le moment déterminant de l'évolution du droit sur cette question est survenu en 1992, à la suite de deux décisions de la Cour supérieure du Québec lesquelles, bien qu'elles n'aient pas été portées en appel, n'en ont pas moins redéfini et cristallisé l'état du droit sur la question. Depuis ce moment, la doctrine juridique et la jurisprudence ont été unanimes à reconnaître le bien-fondé des principes élaborés dans ces deux décisions (Barreau du Québec, 2010, p. 89).

L'une de ces deux décisions est celle de Nancy B. c. Hôtel-Dieu de Québec ${ }^{8}$, dont les médias ont fait grand état. La seconde décision, moins connue du public est celle de Manoir de la Pointe-Bleue c. Corbeil $l^{9}$. Nous ne décrirons pas de façon détaillée les faits et les enjeux juridiques se rapportant à ces deux affaires. Nous nous limiterons à en faire ressortir certaines conclusions générales. Une personne majeure et apte à consentir détient le droit de refuser un traitement, même si ce refus entraînera son décès. Le droit de refus s'exerce à l'égard de toutes les formes de traitement, incluant l'assistance respiratoire, ainsi que l'alimentation et l'hydratation. L'article 217 du Code criminel oblige le médecin à poursuivre un traitement 
entrepris si la cessation de celui-ci «met ou peut mettre la vie humaine en danger». La volonté du patient de cesser un traitement marque la limite de l'obligation du médecin à poursuivre cette intervention. Cette évolution de notre droit offre une réponse à la proposition de la CRD suggérant l'addition au Code criminel d'un texte établissant que le médecin n'a pas l'obligation d'administrer un traitement lorsque le patient le refuse. Quant aux soins palliatifs susceptibles de raccourcir l'espérance de vie du patient, il faut d'abord remarquer que des soins palliatifs adéquats peuvent augmenter l'espérance de vie du patient. Dans l'éventualité où des soins palliatifs adéquats auraient pour effet de raccourcir l'espérance de vie, nous devons prendre en compte aussi bien la qualité que la quantité de vie. Les éléments centraux dans ces circonstances sont le bien-être du patient, et le consentement du patient ou de ses proches, suivant la condition du patient.

\section{LE CONSENTEMENT \\ À SA PROPRE MORT}

Comme nous l'avons indiqué, la première disposition du projet de loi C-384 modifie l'article 14 du Code criminel en autorisant certaines exceptions énoncées dans le projet de loi. Ces exceptions se rapportent au contexte de la personne qui demande à un médecin de l'aider à «mourir dignement». Cette modification de l'article 14 est absolument requise pour la mise en œuvre du «mourir dignement », puisque la personne souffrante qui formule la demande à un médecin exprime un consentement à l'égard d'une intervention qui lui procurera la mort.

L'article $14 \mathrm{du}$ Code criminel est d'une portée générale, qui ne vise pas particulièrement les personnes souffrantes susceptibles de souhaiter mourir. L'article 14 cible plus communément des contextes d'infractions criminelles dans lesquels une personne consentirait à sa propre mort sous la contrainte d'une violence ou d'une manipulation quelconque. En effet, dans le contexte criminel usuel, il serait absurde qu'un meurtrier puisse invoquer le consentement de sa victime comme moyen de défense. Il existe aussi un autre fondement - moins pragmatique - au contenu de l'article 14: un fondement de principe. Chez les théologiens, la vie est considérée comme un don de Dieu qui nous l'a confiée. Avons-nous le droit d'en disposer selon notre volonté ${ }^{10}$ ? La réponse est négative pour celui qui perçoit la vie comme un don déposé en notre possession et à l'origine de certaines obligations destinées à sa sauvegarde.

Le roman Les souliers de Saint Pierre contient une illustration saisissante de cette idée de la vie humaine reçue à titre de don et à laquelle seul Dieu peut mettre un terme. L'histoire se déroule dans les années 1980. L'archevêque ukrainien Cyrille Lakota est libéré après avoir été prisonnier politique en Sibérie pendant 17 ans. Nommé cardinal peu après sa libération, il participe au conclave prochain qui l'élit pape. Le passage qui suit cite des propos de Cyrille I $^{\text {er }}$ lors d'un entretien privé, alors qu'il relate un souvenir de son séjour en prison:

Lorsqu'en Russie je fus jeté en prison pour la première fois, c'était à une époque terrible de tortures et de cruautés. Une nuit, on amena dans ma cellule un homme qui avait été plus abominablement brisé que tous ceux que j'avais vus jusque-là. Il souffrait le martyre, hurlait et suppliait qu'on le tuât pour mettre fin à ses souffrances. Je vous demande en grâce de me croire, la tentation a été forte. Contempler, impuissant, un tel calvaire, cela dégrade et cela terrifie le plus insensible [...] C'est pourquoi je peux comprendre, sans pour autant l'excuser, votre ami médecin. En pareil cas, on a presque l'illusion, en donnant la mort, de collaborer avec la miséricorde divine. Seulement nous ne sommes pas Dieu, et il ne nous appartient pas de décider de la vie et de la mort» (West, 1978, p. 169).

Le souvenir du pape contient deux éléments que nous prenons en considération dans nos discussions sur l'euthanasie et l'aide au suicide: une souffrance intolérable et la volonté de la personne de mourir. Dans le cas du prisonnier, ses tortionnaires semblent peu disposés à lui administrer des soins palliatifs. Néanmoins, pour Cyrille, la position de principe demeure: nous ne sommes pas Dieu.

Le philosophe anglais John Locke (1632-1704) expose un point de vue semblable: "Nobody can give more power than he has himself; and he that cannot take away his own life, cannot give another power over it» (Locke, 1824: 143). Tout homme détient la possibilité matérielle de s'enlever la vie. Mais peut-il, de façon morale, autoriser une autre personne à la lui enlever? Pour Locke, l'humain, ne disposant pas du droit de mettre un terme à sa vie, ne peut céder ce droit à un autre. La volonté de mourir de l'un n'autorise donc pas l'autre à l'aider dans la réalisation de son intention.

\section{LES DOULEURS AIGUËS}

Chacun peut comprendre qu'une personne atteinte depuis plusieurs années d'une maladie débilitante et douloureuse demande à «mourir dignement » après avoir essayé sans succès les interventions thérapeutiques disponibles. Cependant, la demande de «mourir dignement» relève d'une autre perspective lorsqu'elle provient d'une personne malade depuis peu de temps ou qui a refusé de recevoir les traitements adéquats. Une jeune femme dans la vingtaine atteinte d'anorexie nerveuse depuis une dizaine d'années demande à mourir dignement, épuisée par sa maladie, rebutée par ses conflits incessants avec ses proches et son médecin, anéantie devant la perspective d'être de nouveau hospitalisée. Dans un autre cas, un homme dans la quarantaine en a assez de lutter contre une dépression qui semble réfractaire à tout traitement. Une dernière illustration pourrait être celle d'une dame à qui son médecin vient d'apprendre qu'elle est atteinte de sclérose latérale amyotrophique, et qui refuse de se voir progressivement diminuer durant plusieurs années. Suivant le dispositif du projet de loi, toutes ces personnes seraient admissibles à «mourir dignement». Par contre, leurs situations respectives diffèrent beaucoup l'une de l'autre et, dans chaque cas, la demande de «mourir dignement» soulève de graves questions de nature éthique.

Le document de la CRD envisage la possibilité que quelqu'un profite d'un état de faiblesse chez une personne pour l'inciter à se suicider: "Que dire de celui qui, profitant de l'état dépressif d'une autre personne, la pousse au suicide pour en tirer un bénéfice pécuniaire» (Commission de réforme du droit du Canada, 1982: 61)? L'hypothèse que pose la CRD est celle d'un comportement effrayant et criminel. Il faudra évaluer si le dispositif de tout projet de loi sur le «mourir dignement» protège les personnes vulnérables contre de tels agissements.

\section{LE FORUM DE DISCUSSION}

En 1993, la Cour suprême du Canada a été saisie d'une affaire portant sur l'aide au suicide ${ }^{11}$. Âgée de 42 ans, madame Sue Rodriguez est atteinte de sclérose latérale amyotrophique, une maladie débilitante incurable. Son espérance de vie se situe entre 2 et 14 mois. Madame Rodriguez demande au tribunal d'émettre une ordonnance autorisant un médecin à aménager les instruments techniques qui lui permettraient de se donner elle-même la mort au moment qu'elle choisirait. Le mémoire du Barreau du Québec sur les soins de fin de vie décrit l'attente de madame Rodriguez et la décision de la Cour suprême:

La condition physique de Mme

Rodriguez se détériorait rapidement.

Elle serait bientôt incapable

d'avaler, de parler, de marcher et 
de bouger sans aide. Elle n'aurait plus la capacité de respirer sans respirateur, de manger sans subir de gastrotomie et serait finalement alitée en permanence. Mme Rodriguez souhaitait décider des circonstances, des conditions et du moment de sa mort. Elle ne souhaitait pas mourir tant qu'elle pouvait jouir de la vie, mais compte tenu qu'au moment où elle perdrait ses capacités de jouir de la vie, elle serait physiquement incapable d'y mettre fin sans assistance, elle désirait que la Cour lui permette d'obtenir l'aide nécessaire.

Cette décision soulevait donc directement l'application de l'article 241 du Code criminel. Le jugement de la Cour suprême du Canada dans cette affaire, fut rendu à cinq juges contre quatre. Ce résultat assez serré reflète bien les incertitudes de la société canadienne de cette époque et ne permet pas de conclure que la question a été définitivement réglée par ce jugement (Barreau du Québec, 2010, p. 36).

Il n'est pas déraisonnable de penser qu'une décision rendue à cinq juges contre quatre pourrait être différente aujourd'hui. Par contre, nous ne pouvons pas affirmer avec certitude que les progrès des soins palliatifs et l'évolution des mentalités généreraient une décision différente si la question revenait devant la cour.

Le rapprochement de ces deux événements, la décision rendue dans l'affaire Sue Rodriguez et le dépôt du projet de loi C-384, nous interpelle à propos de la question de savoir quel est le forum approprié pour décider de l'acceptabilité de l'euthanasie et de l'aide au suicide. Du point de vue de la légitimité et de la compétence de nos institutions, une telle décision devraitelle être celle de la Cour suprême ou celle du Parlement?

Le professeur de droit Steven G. Calabresi demande pourquoi les citoyens d'une démocratie devraient permettre à un comité formé d'avocats d'adopter des décisions contraignantes concernant les questions morales et religieuses les plus sensibles, par un vote de cinq contre quatre basé sur les croyances morales et religieuses des membres de ce comité ${ }^{12}$. Ce propos vise évidemment les juges de la Cour suprême des États-Unis. Le même commentaire pourrait s'adresser aux juges de la Cour suprême du Canada, particulièrement dans le contexte de l'affaire Rodriguez décidée à cinq contre quatre. Selon le professeur Calabresi, demander aux juges d'instaurer des politiques publiques n'est pas pragmatique, puisque les juges ne sont pas qualifiés pour adopter des politiques publiques ni pour évaluer les conséquences de leurs décisions prises au cas par cas. Les juges disposent de moins d'informations que les élus, qui peuvent tenir des auditions publiques, discuter avec leurs électeurs et consulter des experts.

La critique du professeur Calabresi à l'égard des juges semble excessive. Si les juges se prononcent suivant leurs croyances morales et religieuses, le même commentaire s'adresse aux membres du Congrès et à ceux du Parlement. Par ailleurs, la tenue d'un procès équitable contraint les tribunaux à considérer uniquement la preuve et l'argumentation déposées devant eux. Enfin, il arrive que les cours de justice doivent trancher dans des matières faisant l'objet de débats publics, alors que la loi est dépassée par l'évolution de l'esprit de la nation, et que, pour des motifs reliés à la politique partisane, les élus craignent d'intervenir. y a entre envisager l'euthanasie du point de vue légal et l'envisager du point de vue éthique.

Publié en 1982, le document de la CRD veut explorer certaines questions fondamentales de politique sociale dans l'intention d'encourager la tenue d'un débat public. Un tel débat doit précéder une éventuelle réforme légale, qui représenterait la manifestation juridique de certains choix sociaux à propos desquels se dégagerait un consensus plus ou moins partagé.

Le document de la CRD rappelle que la vie humaine constitue une valeur fondamentale des systèmes de droit modernes. Toutefois, un nouveau courant de pensée est apparu dans les pays occidentaux. La vie demeure une valeur fondamentale, mais cette reconnaissance est tempérée par une préoccupation pour la qualité de vie. Ainsi, le développement des soins palliatifs permet de cesser les interventions

IL N’EST PAS DÉRAISONNABLE DE PENSER QU'UNE DÉCISION RENDUE À CINQ JUGES CONTRE QUATRE POURRAIT ÊTRE DIFFÉRENTE AUJOURD'HUI. PAR CONTRE, NOUS NE POUVONS PAS AFFIRMER AVEC CERTITUDE QUE LES PROGRÈS DES SOINS PALLIATIFS ET L'ÉVOLUTION DES MENTALITÉS GÉNÉRERAIENT UNE DÉCISION DIFFÉRENTE SI LA QUESTION REVENAIT DEVANT LA COUR.

Toutefois, la thèse du professeur Calabresi nous rappelle que le Parlement est le forum à privilégier pour tenir une discussion aussi considérable que celle portant sur l'euthanasie et l'aide au suicide. Montesquieu (1689-1755) affirme que «C'est au législateur à suivre l'esprit de la nation, lorsqu'il n'est pas contraire aux principes du gouvernement; car nous ne faisons rien de mieux que ce que nous faisons librement, et en suivant notre génie naturel»(Montesquieu, 1973: 330). Le dépôt du projet de loi C-384 ou de tout autre projet de loi de même nature fournit au législateur l'occasion de s'enquérir de l'esprit de la nation, à la condition qu'il pousse son investigation au-delà de la deuxième lecture.

Pour être sensé, un débat portant sur l'euthanasie et l'aide au suicide doit respecter certains préalables: nous devons nous entendre sur une définition de l'euthanasie; nous devons refuser d'éviter la circonstance limite dans laquelle se trouve la personne que les soins palliatifs ne soulagent pas, et qui continue à demander qu'on l'aide à mourir; enfin, nous devons prendre conscience de la différence qu'il thérapeutiques et de dispenser des soins destinés à assurer le meilleur confort possible en phase terminale.

Au moment de la publication du document de la CRD, plusieurs médecins souhaitent être guidés par un énoncé précis de leurs obligations légales, plutôt que de dépendre de la discrétion en matière de poursuite qu'exerce la Couronne. Cette discrétion est génératrice d'une incertitude qui ne rend service ni aux médecins ni à leurs patients. Au terme de sa réflexion, la CRD formule certaines recommandations que nous avons exposées de façon détaillée: prohibition de l'euthanasie active, refus de la décriminalisation de l'aide au suicide, reconnaissance de la légalité de l'interruption de traitement et de la renonciation au traitement.

Vingt-huit ans après la publication du document de la CRD, le projet de loi C-384 veut autoriser l'intervention d'un médecin pour aider une personne âgée d'au moins 18 ans à «mourir dignement». Un médecin peut aider une personne à "mourir dignement» si la personne «continue, après avoir essayé ou expressément refusé les traitements appropriés et dispo- 
nibles, d'éprouver des douleurs physiques ou mentales aiguës sans perspective de soulagement» ou si elle est atteinte d'une maladie en phase terminale.

Le document de la CRD valorise considérablement la conjonction entre l'exercice de l'autonomie personnelle et l'appréciation de la qualité de vie. Pour la CRD, la personne concernée procède à une appréciation de sa qualité de vie et décide des soins qu'elle recevra ou pas. Le projet de loi C-384 est fondé sur un mouvement similaire: la personne qui exprime sa volonté de «mourir dignement » a apprécié sa qualité de vie présente ou éventuelle, et elle exerce son autonomie en demandant à mourir avec l'aide d'un médecin.

Le projet de loi C-384 place le droit de choisir, l'exercice de l'autonomie personnelle au sommet des valeurs, et en fait son paradigme. Suivant le paradigme de l'exercice de l'autonomie personnelle, la personne peut légitimement demander de «mourir dignement». Le document de la CRD attribue lui aussi une grande importance à l'exercice de l'autonomie personnelle, mais il estime que la décriminalisation de l'aide au suicide n'est pas acceptable en raison des dangers d'abus qu'elle comporte.

Plusieurs éléments de discussion contenus dans le document de la CRD demeurent pertinents dans les débats actuels, notamment les références axiologiques que constituent les principes primordiaux: la préservation de la vie humaine comme valeur fondamentale, l'autonomie et l'autodétermination de la personne, la légitimité des considérations portant sur la qualité de vie, et la protection particulière des personnes vulnérables. Ces principes doivent être soupesés de nouveau à la lumière des progrès de la médecine et de l'évolution des mentalités. Quant aux préoccupations reliées au droit à la cessation de traitement, la jurisprudence semble y répondre de façon adéquate depuis 1992.

Bien que défait en seconde lecture, le projet de loi C-384 demeure une contribution majeure au débat public sur l'euthanasie et l'aide au suicide. Un élément de ce projet de loi est particulièrement audacieux: l'ouverture du «droit de mourir dignement» à une personne qui ne se trouve pas en phase terminale, mais qui ressent «des douleurs physiques ou mentales aiguës sans perspective de soulagement». Cette ouverture fait entrer dans un autre lieu que celui de la fin de vie les possibilités de la conjonction entre l'exercice de l'autonomie personnelle et l'appréciation de la qualité de vie. Pour la CRD, la notion de "mort dans la dignité» s'applique au patient qui «doit pouvoir exercer un choix constant sur la façon dont il entend mourir et surtout sur la façon dont il entend vivre ses derniers moments » (Commission de réforme du droit du Canada, 1982: 64). Il n'y est nullement question de la «mort dans la dignité » pour une personne qui souffre, mais qui ne se trouve pas en fin de vie.

Un jour, un parlementaire canadien déposera un projet de loi semblable au projet de loi C-384. Ce parlementaire devra préparer une argumentation qui répond à deux objections. Premièrement, la vie est porteuse d'un caractère sacré dont la symbolique influence l'ensemble de notre conduite. Accepter qu'on puisse légalement mettre un terme à la vie d'une personne aura pour effet d'entamer la transcendance de notre idéal à l'égard du respect que nous devons accorder à la vie humaine. Deuxièmement, aucune loi proclamant le «droit de mourir dignement» n'est suffisamment étanche pour protéger entièrement les personnes vulnérables contre les errements et les excès.

\section{Bibliographie}

BARREAU DU QUÉBEC (2010). Pour des soins de fin de vie respectueux des personnes, Montréal.

BEAUCHAMP, T.L., et CHILDRESS, J.F. (2009). Principles of Biomedical Ethics, $6^{\mathrm{e}}$ édition, New York, Oxford University Press.

BERLIN, I. (1958). "Two Concepts of Liberty.» In Isaiah Berlin (1969), Four Essays on Liberty, Oxford, Oxford University Press, p. 2.

BLAIS, M. (2000). Le Chien de Socrate, Chicoutimi, Les éditions JCL.

CALABRESI, S.G. (2007). Originalism: A Quarter-century of Debate, Foreword by Justice Antonin Scalia, Washington, D.C., Regnery Publishing

COMMISSION DE RÉFORME DU DROIT DU CANADA (1982). Euthanasie, aide au suicide et interruption de traitement, Ottawa, ministre des Approvisionnements et Services.

DENDIEN, J. (2012). Le Trésor de la Langue Française informatisé. Disponible: $<$ http://atilf.atilf.fr/dendien/scripts/tlfiv5/ advanced.exe ?8;s=2005441125>

DICTIONNAIRES LE ROBERT (1998). Dictionnaire historique de la langue française, Paris, Tome 1.

FÉDÉRATION DES MÉDECINS OMNIPRATICIENS DU QUÉBEC (2010). Mémoire à la Commission spéciale sur la question de mourir dans la dignité.

FÉDÉRATION DES MÉDECINS SPÉCIALISTES DU QUÉBEC (2010). Droit de mourir dans la dignité. Mémoire présenté à la Commission de la santé et des services sociaux (Mise à jour: août 2010)
FRÉCHETTE, L. (2005). Être ou ne pas être branché, là est la question dans Repères, Droit civil en ligne (DCL), EYB2005REP358.

KÜNG, H. (1985). Vie éternelle? Paris, Seuil.

LALONDE, F. (2009). Débats de la Chambre des communes - Hansard, le vendredi 2 octobre 2009, Ottawa. Disponible: $<$ http:// www.parl.gc.ca/HousePublications / Publication.aspx ? DocId $=4122372 \&$ Lan guage $=\mathrm{F} \& \operatorname{Mode}=1 \& \operatorname{Parl}=40 \& \mathrm{Ses}=2 \#$ OOB-2876558>

LALONDE, F. (2010). Débats de la Chambre des communes - Hansard, le mardi 20 avril 2010, Ottawa. Disponible: <http:// www.parl.gc.ca/HousePublications/ Publication.aspx ? Language $=\mathrm{F} \&$ Mode $=1$ $\&$ Parl $=40 \&$ Ses $=3 \&$ DocId $=4446673 \&$ Fil $\mathrm{e}=0$ \#OOB-3107357>

LOCKE, J. (1824). Two Treatises of Government. Second Treatise of Civil Government, Londres, Harvard College Library.

MANOIR DE LA POINTE-BLEUE c. CORBEIL, 1992, R.J.Q. 712.

MONTESQUIEU, C. (1973) De l'esprit des Lois, Tome 1, Paris, Garnier.

NANCY B. c. Hôtel-Dieu de Québec, 1992, R.J.Q. 361.

RODRIGUEZ c. Colombie-Britannique (Procureur général), (1993) 3 R.C.S. 519.

SÉNAT DU CANADA (1995). De la vie et de la mort. Rapport du Comité sénatorial spécial sur l'euthanasie et l'aide au suicide, Ottawa, ministre des Approvisionnements et Services.

SHAKESPEARE, W. (1959). Beaucoup de bruit pour rien. (Much Ado About Nothing), Euvres complètes. Paris, Pléiade.

THOMAS D'AQUIN (1986). Somme théologique, tome 4, Paris, Les éditions du Cerf.

VOYER, G. (2009). La mort à son heure, Montréal, Médiaspaul.

WEST, M. (1978). Les souliers de Saint Pierre, Paris, Librairie Plon.

\section{Notes}

1. «Plusieurs personnes confondent régulièrement le refus et la cessation de traitement, l'acharnement et l'abandon thérapeutiques, la sédation continue ou terminale et l'euthanasie. Ne serait-il pas possible, qu'après discussion, ces personnes fassent la part des choses et entrevoient alors différemment l'idée même de l'euthanasie, ne l'estimant plus nécessaire pour cheminer vers la mort dans la dignité? Cela met en relief l'important besoin d'information et d'éducation de la population à cet égard et l'importance déterminante d'un dialogue entre l'équipe traitante et la personne ainsi que sa famille quant aux choix de traitements disponibles. » Fédération des médecins omnipraticiens du Québec (2010). Mémoire à la Commission spéciale sur la question de mourir dans la dignité, p. 9.

2. "Withholding or withdrawing treatment will hasten death only for those individuals who could be or are being sustained by a technology. Many other individuals, including some 
patients with cancer, face a protracted period of dying when respirators and other life-preserving technology are not being utilized. Great improvements in and extensions of palliative care adequately address the needs of many, perhaps most, of these patients. However, for some of these patients, palliative care and the ability to refuse treatment do not adequately address all their concerns. During their prolonged period of dying, they may endure a loss of functional capacity, unremitting pain and suffering, an inability to experience the simplest of pleasures, and long hours aware of the hoplessness of their condition. Some patients find this prospect unbearable and desire a painless means to hasten their deaths. " Beauchamp, T.L. et Childress, J.F. (2009). Principles of Biomedical Ethics, $6^{\mathrm{e}}$ édition, New York, Oxford University Press, p. 176.

3. «En fin de vie, la transgression de l'interdit de l'homicide, c'est l'euthanasie. Je ne peux écarter la possibilité qu'un jour ou l'autre se présente une situation où, par exemple, la douleur soit véritablement incontrôlable et où l'on puisse considérer comme plus humain d'abréger intentionnellement la vie du malade. Je doute qu'il y ait beaucoup de situations à ce point intolérables qu'elles justifient de prendre un tel risque moral, mais je ne peux l'exclure totalement. [...] Je considère donc que la possibilité d'une transgression légitime de l'interdit de l'homicide ne justifie en rien la légalisation de l'euthanasie. » Voyer, G. (2009). La mort à son heure, Montréal, Médiaspaul, p. 51.

4. "To coerce a man is to deprive him of freedom - freedom from what? Almost every moralist in human history has praised freedom. Like happiness and goodness, like nature and reality, it is a term whose meaning is so porous that there is little interpretation that it seems able to resist. I do not propose to discuss either the history of this protean word or the more than two hundred sensesof it recorded by historians of ideas. I propose to examine no more than twoof these senses but they are central ones, with a great deal of human history behind them, and, I dare say, still to come. The first of these political senses of freedom or liberty (I shall use both words to mean the same), which (following much precedent) I shall call the "negative" sense, is involved in the answer to the question "What is the area within which the subjecta person or group of persons - is or should be left to do or be what he is able to do or be, without interference by other persons?" The second, which I shall call the "positive" sense, is involved in the answer to the question "What, or who, is the source of control or interference that can determine someone to do, or be, this rather than that?" The two questions are clearly different, even though the answers to them may overlap. " Berlin, I. (1958). «Two Concepts of Liberty » In Isaiah
Berlin (1969) Four Essays on Liberty. Oxford, Oxford University Press, p. 2.

5. "Justifying an act is distinct from justifying a practice or a policy that permits or even legitimates the act's performance. A rule of practice or a public policy or a law that prohibits various forms of assistance in dying in medicine may be justified even if it excludes some acts of causing a person's death that in themselves are morally justifiable. For example, there may be sufficient reasons that a law might not permit physicians to use a drug overdose to cause death for a patient who suffers from terrible pain, who will probably die within a few weeks, and who requests a merciful assisted death. However, this same act might be morally justified in an individual case. " Beauchamp, T.L. et Childress, J.F. (2009). Principles of Biomedical Ethics, 6e édition, New York, Oxford University Press, p. 177.

6. "Le suivi des patients atteints de maladies chroniques apporte son lot de problématiques particulières en termes d'intensité de soins puisque les problèmes souvent complexes des patients ne peuvent que s'aggraver. Dans de telles circonstances, il se crée souvent des attentes démesurées envers la médecine. Ces situations requièrent beaucoup d'empathie de la part du médecin, et ce dernier doit faire preuve de flexibilité dans son offre de traitement et d'accompagnement. Ce type de suivi oblige aussi un partage des responsabilités, une complémentarité entre les médecins spécialistes, les médecins généralistes et autres intervenants. » Fédération des médecins spécialistes du Québec (2010). Droit de mourir dans la dignité. Mémoire présenté à la Commission de la santé et des services sociaux, (Mise à jour : août 2010), p. 5.

7. «Les tribunaux ont eu diverses occasions de se pencher sur des cas où s'opposaient le droit à l'euthanasie, le droit au suicide assisté et le droit au refus de soins. Pensons aux affaires Nancy B., Sue Rodriguez, Latimer et Manoir de la Pointe Bleue c. Corbeil. Dans toutes ces causes, seul le droit au refus de soins a été reconnu. Ainsi, bien que le refus de soins puisse mener son auteur à la mort, il est valable lorsqu'il s'agit d'une décision libre et prise en toute connaissance de cause. » Fréchette, L. (2005). Être ou ne pas être branché, là est la question dans Repères, Droit civil en ligne (DCL), EYB2005REP358.

8. Nancy B. c. Hôtel-Dieu de Québec, 1992, R.J.Q. 361.
9. Manoir de la Pointe-Bleue c. Corbeil, 1992, R.J.Q. 712.

10. «La discussion théologique cherche à savoir si l'homme a le droit de disposer de sa vie jusques et y compris sa mort. Notons-le bien, la question se pose ici pour nous non à propos de bien-portants, mais de malades graves (moribonds). Je ne parle pas ici de quelqu'un qui souffre simplement d'un mal de vivre souvent passager d'ailleurs -, comme par exemple d'un jeune homme dont le premier amour a été rompu et qui doute à présent de la vie. Non, je parle de quelqu'un qui, au terme de sa vie, souffre d'une maladie incurable, et qui va bientôt affronter la mort. Un tel homme a-t-il le droit de disposer de sa vie? » Küng, H. (1985). Vie éternelle? Paris, Seuil, p. 232.

11Rodriguez c. Colombie-Britannique (Procureur général), (1993) 3 R.C.S. 519.

12 «Wy on Earth should the citizens of a democracy allow a committee of superannuated lawyers to make binding rules on the most sensitive issues of morality and religion on a five-to-four vote based on their own personal moral and religious beliefs? Why especially should we combine this with an ordinary lawmaking system in which laws implementing mundane matters like trucking deregulation must pass two houses of Congress, overcome a filibuster in the Senate, and be either signed by the President or repassed by a two-thirds vote of both houses?

Telling judges to be policy makers is itself unpragmatic and will lead to bad results not good ones. Judges are not good at making policy or judging consequences on a case-bycase basis. They have much less information at their disposal than do legislators because they cannot hold hearings, they cannot visit their home districts and talk to constituents, and they cannot engage in ex parte contacts with experts. " Calabresi, S.G. (2007). Originalism: A Quarter-century of Debate, Foreword by Justice Antonin Scalia, Washington, D.C., Regnery Publishing, Inc., p. 26. 